\title{
HIV Infection of brain macrophages and microglia
} Rebecca Dunfee, Elaine Thomas, Megan Mefford, Petronela Ancuta, Anupa Kamat, Kevin Kunstman, Steven Wolinsky and Dana Gabuzda*

\author{
Address: Department of Cancer Immunology and AIDS, Dana-Farber Cancer Institute and Department of Neurology, Harvard Medical School, \\ Boston, MA, 02115, USA \\ Email: Dana Gabuzda* - dana_gabuzda@dfci.harvard.edu \\ * Corresponding author
}

from Infectious diseases of the nervous system: pathogenesis and worldwide impact

Paris, France. 10-13 September 2008

Published: 23 September 2008

BMC Proceedings 2008, 2(Suppl I):SI I

This abstract is available from: http://www.biomedcentral.com/I753-656I/2/SI/SI I

(c) 2008 Dunfee et al; licensee BioMed Central Ltd.

HIV infects brain macrophages and microglia, and causes HIV-associated dementia (HAD) and milder forms of neurocognitive impairment in $20-30 \%$ of patients who develop AIDS. HIV entry into macrophages and microglia is initiated by interaction between the envelope glycoprotein (Env) surface subunit gp120 and CD4, which induces a conformational change in gp120 that exposes the coreceptor binding site. The interaction of CD4-bound gp120 with a co-receptor, usually CCR5 or CXCR4, triggers conformational changes in gp120 and the transmembrane subunit gp41 that enable fusion and virus entry. CCR5 is the primary co-receptor used for virus entry into macrophages and microglia, but CCR5 usage is neither necessary nor sufficient for macrophage tropism, suggesting that determinants other than those that specify coreceptor usage influence the capacity of HIV to enter macrophages/microglia. Macrophages and microglia express lower levels of CD4 on the cell surface than CD4+ T cells in peripheral blood. By a combination of genetic, molecular, and biochemical approaches, we identified specific amino acid variants in the HIV envelope glycoproteins that increase viral tropism for macrophages/microglia by enhancing gp120-CD4 affinity (i.e., N283 in C2 region of gp120) or exposure of the CD4 binding site (i.e., loss of an N-linked glycan at 386 in V4 region of gp120). These variants were more frequent in the brain of patients who developed dementia compared to nondemented patients, implying the importance of enhance HIV Env-CD4 interactions for HIV neuropathogenesis in vivo. Elevated plasma lipopolysaccharide (LPS), an indicator of micro- bial translocation from a damaged leaky gut, is a likely cause of systemic immune activation in chronic HIV infection. LPS induces monocyte activation and trafficking into brain, which are key mechanisms in the pathogenesis of HAD. To determine whether high LPS levels are associated with increased monocyte activation and HAD, we obtained peripheral blood samples from AIDS patients and examined plasma LPS, peripheral blood monocytes, and soluble markers of monocyte activation. Circulating monocytes expressed high levels of the activation markers CD69 and HLA-DR, and harbored low levels of HIV compared to CD4+ T-cells. High plasma LPS levels were associated with increased plasma sCD14 and LPS-binding protein levels, and low endotoxin core antibody levels. LPS levels were higher in HAD patients compared to control groups, and were associated with HAD independently of plasma viral load and CD4 counts. These results provide a better understanding of mechanisms that underlie neurotropism of HIV and suggest a role for elevated LPS levels in driving monocyte activation and subsequent trafficking of activated monocytes into the CNS, thereby promoting neuroinflammation and increasing availability of target cells permissive for HIV replication in brain. 